\title{
Utilidad del tiempo de protrombina y el tiempo parcial de tromboplastina activada en la valoración preoperatoria de la hemostasia en pediatría
}

\author{
Usefulness of prothrombin and activated partial thromboplastin time in the preoperative assessment of \\ hemostasis in pediatrics
}

Raúl Copana Olmos ${ }^{1}$, Mauricio Peralta Caballero ${ }^{2}$, Roberto Unzueta Quiroga ${ }^{3}$, Gonzalo Carpio Deheza ${ }^{4}$

\section{Resumen}

Para evaluar la hemostasia preoperatoriamente una historia clínica y examen físico dirigidos están indicados, siendo el uso de pruebas de coagulación recomendados solo cuando existe alguna indicación, y no de rutina; Objetivo: el presente estudio pretende conocer la utilidad del TP y APTT en la valoración preoperatorio de coagulopatías en cirugías programadas menores y ambulatorias. Métodos: se realizó un estudio prospectivo, observacional en un hospital quirúrgico terciario; seleccionamos pacientes sometidos a procedimientos menores y ambulatorio, excluyendo aquellos con comorbilidades, riesgo quirúrgico de sangrado alto o con medicación que interfiera con la coagulación. Resultados: se reclutaron 69 pacientes, se aplicó la historia clínica y el examen físico dirigido identificando 1 paciente sospechoso de trastorno de coagulación (posteriormente descartado); Se realizaron 218 exámenes complementarios: 69 rutinarios (TP, APTT, hemograma) y 149 no rutinarios (Indicados de forma arbitraria), obteniendo valores medios en rangos normales y no pudiendo identificar o descartar trastornos de coagulación con ellos, pero observando un $21 \%$ (15 casos) resultados anormales, lo que adicionalmente ocasiono conductas para confirmar o corregir estos valores, que van desde repetir la prueba a transfundir hemoderivados; generando un costo promedio global de 102 Bs. por paciente, sin un beneficio o cambio en la conducta clínica o quirúrgica, Conclusión: el estudio estableció que las pruebas rutinarias de screening preoperatorio tienen poca utilidad y son poco costo-beneficiosas en la valoración de la hemostasia para procedimientos menores o ambulatorios, en comparación de una historia clínica y examen físico dirigido; siendo apropiada su indicación cuando existan hallazgos anormales en el examen físico e historia clínica o en base a enfermedades concomitantes.

Palabras clave: screening, hemostasia, cirugía pediátrica, valoración preoperatoria

\section{Abstract}

To evaluate hemostasis preoperatively, a directed clinical history and physical examination are indicated, and the use of routine coagulation being recommended when there is some indication, and not routine; Objective: the present study aims to know the usefulness of PT and APTT in the preoperative assessment of coagulopathies in scheduled minor and outpatient surgeries. Methods: A prospective, observational study was conducted in a tertiary surgical hospital; We select patients undergoing minor and outpatient procedures, excluding those with comorbidities, surgical risk of high bleeding, or with medication that interferes with coagulation. Results: 69 patients were recruited, the clinical history and the directed physical examination were applied, identifying 1 patient suspected of coagulation disorder (later discarded); 218 complementary tests were performed: 69 routine (PT, APTT, blood count) and 149 non-routine (arbitrarily indicated), obtaining mean values in normal ranges and not being able to identify or rule out coagulation disorders with them, but observing $21 \%$ ( 15 cases) abnormal results (false positives), which additionally led to behaviors to confirm or correct these values, ranging from repeating the test to transfusing blood products; generating a global average cost of $102 \mathrm{Bs}$. per patient, without a benefit or change in clinical or surgical behavior. Conclusion: the study established that routine preoperative screening tests have little utility and are little cost-beneficial in the assessment of the hemostasis for minor or outpatient procedures, compared to a history and directed physical examination; its indication being appropriate when there are abnormal findings in the physical examination and clinical history or based on concomitant diseases.

Keywords: screening, hemostasia, pediatric surgery, preoperative assesment

${ }^{1}$ Terapista Intensivo Pediátrico. Responsable Servicio de Terapia Intermedia, Hospital del Niño Manuel Ascencio Villarroel; Docente Investigador Posgrado, Universidad Mayor de San Simón; Sociedad Boliviana de Terapia Intensiva Pediátrica.

https://orcid.org/0000-0002-3084-7611.

${ }^{2}$ Traumatologo. Jefe del departamento de Cirugia Pediatrica, Hospital del Niño Manuel Ascencio Villarroel. https://orcid.org/0000-0002-5664-4281. ${ }^{3}$ Medico Salubrista. Departamento de planificación, Escuela Técnica de Salud. Ministerio de Salud Bolivia. https://orcid.org/0000-0001-5823-2419. ${ }^{4}$ Cirujano General. Subdirector Médico, Hospital Clínico Viedma; Docente Investigador Posgrado, Universidad Mayor de San Simón. https://orcid.org/0000-0001-0822-1567

${ }^{*}$ Correspondencia a: Raul Copana O. MD.

Correo electrónico: dr_copana_raul@yahoo.com

Recibido el 24 de agosto de 2020. Aceptado el 30 de octubre de 2020.
T a patología quirúrgica en pediatría representa una causa Lfrecuente de atenciones en la práctica cotidiana. En la actualidad es aceptado en caso de programar una cirugía menor o un procedimiento ambulatorio, la solicitud de exámenes complementarios a modo de tamizaje de trastornos de coagulación solo en casos en los que se identifique antecedentes familiares de coagulopatía o una historia clínica y examen físicos sugerentes de trastorno de la coagulación, o si la información genética sobre la familia biológica no es evaluable ${ }^{1-3}$. El nivel de evidencia que sustenta estas recomendaciones se basa en estudios observacionales, pero 
no en ensayos clínicos aleatorios, lo cual genera controversia a la hora de aplicar estas recomendaciones especialmente en establecimientos con recursos limitados y en poblaciones con alta prevalencia de anemia y desnutrición, lo que podría repercutir y de cierta forma diferir de la población estudiada en los mencionados estudios ${ }^{4-15}$. Conocer estos datos aportaran información útil al momento de la evaluación preoperatoria pediátrica y permitirá la toma de decisiones costo/beneficiosas para los pacientes y la institución.

El presente estudio tiene el propósito de conocer la utilidad del TP y APTT en el screening preoperatorio de coagulopatías en niños sometidos a intervenciones quirúrgicas ambulatorias o cirugías programadas menores en el departamento de cirugía del Hospital del Niño Manuel Ascencio Villarroel (HNMAV)

\section{Material y métodos}

Se diseñó un estudio unicéntrico, prospectivo, observacional en el área de cirugía pediátrica del HNMAV, único hospital del sector público de 3er nivel de complejidad con este servicio, se estableció un tamaño muestra de 69 paciente, considerando un margen de error del 5\%, y un $95 \%$ confianza para un universo finito de 321 cirugías menores y programadas; fueron reclutados desde enero del 2019 hasta completar la muestra en junio 2020. Fueron incluidos al estudio pacientes sin comorbilidades previas (hepáticas, hematológicas, oncológicas o nutricionales conocidas), con procedimientos a realizarse ambulatorios o cirugías menores, riesgo de sangrado estimado bajo, sin medicación que interfiera con la coagulación.

Las recolecciones de los datos se realizaron en dos tiempos:

- Tiempo 1: Durante la evaluación pediátrica preoperatoria en consulta externa o el primer día de internación, se realizaron las siguientes mediciones:

- Llenado del cuestionario sugerido por Rapapport $^{1}$

- Busqueda y registro de hallazgos clínicos sugeridos por $\mathrm{Chee}^{2}$

- Solicitud y registro de resultados de exámenes TP, APTT y plaquetas y otros sugeridos por el cirujano tratante o el equipo de anestesiología.

- Tiempo 2: Periodo postoperatorio temprano y tardío

- Registro de complicaciones hemorrágicas tempranas (horas) y tardías (días) posteriores a la cirugía

- Registro de costos de laboratorios, medicación y hemoderivados consumidos por el paciente, para corregir alteraciones del coagulograma.

Los datos fueron analizados con el programa IBM statisttics SPSS v25, realizandose los estadígrafos descriptivos de tendencia central y un análisis bivariado de correlación lineal entre las variables estudiadas y los resultados (outcomes hospitalarios y los costos).

El estudio fue aprobado por el comité de bioética del HNMAV en octubre del 2018, al tratarse de un estudio descriptivo sin intervención en humanos, se obvió del uso

Grafico 1. Flujograma de selección de los pacientes del estudio
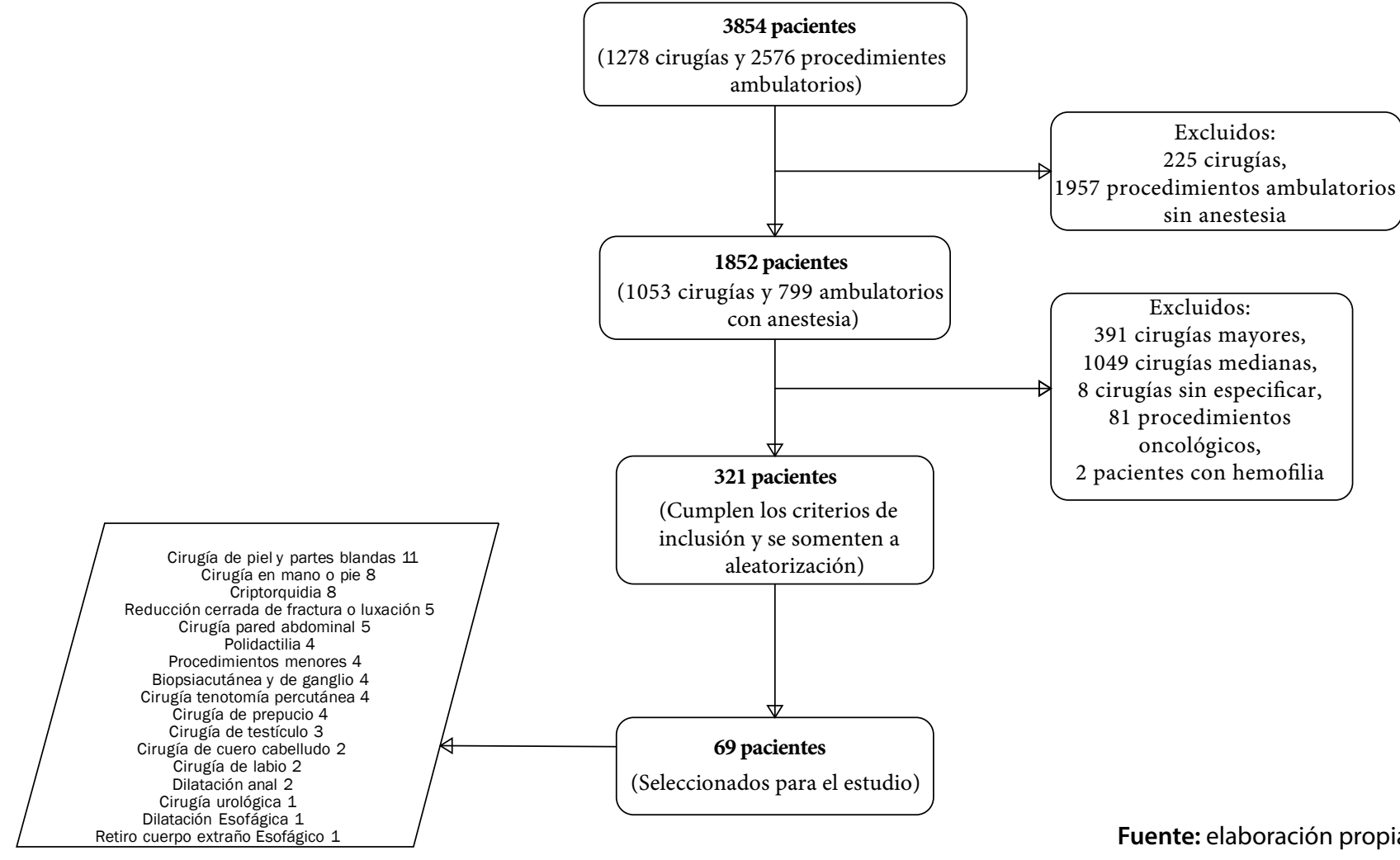
sin anestesia

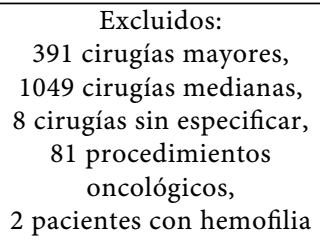

Fuente: elaboración propia. 
de un consentimiento informado específico, se guardaron en cuenta todas las consideraciones éticas sugeridas por el comité.

\section{Resultados}

El estudio reclutó un total de 69 pacientes que cumplían con los criterios de inclusión y exclusión para el estudio, evidenciándose una variedad de distintos procedimientos ambulatorios y quirúrgicos menores dentro de la muestra (Gráfico 1).

Todos los pacientes fueron evaluados clínicamente por un pediatra, el cual aplico los cuestionarios y el examen físico dirigido a la valoración de la hemostasia, encontrando 1 paciente sospechoso de coagulopatía (1,5\% de los casos), sin embargo, dicho caso tuvo los resultados de los exámenes complementarios normales, no presentó sangrados intra o postoperatorios, ni fue diagnosticado de una coagulopatia posteriormente(Tabla 1).

En 69 pacientes se realizaron 218 exámenes complementarios (69 rutinariamente y 149 no rutinariamente), en los pacientes en los que se solicitaron exámenes no rutinarios los más solicitados fueron la creatinina $47,8 \%$, Urea $36,2 \%$, Glicemia $26,1 \%$ Na y $\mathrm{K} 17 \%, \mathrm{Cl}$ y Ca $14,5 \%$, GOT y GPT 11,5\%, Tiempo de coagulación $18,8 \%$ y tiempo de sangría $2,9 \%$; el motivo de dichas solicitudes se basa principalmente en el criterio del cirujano tratante o del anestesiólogo que asistirá el procedimiento, vale mencionar que ningún paciente tiene comorbilidades o patología de base conocidas en la historia clínica y que solo en 1 caso se evidencio al examen físico un hallazgo sugerente de coagulopatía, siendo los demás pacientes sin indicación pediátrica que justifique clínicamente la solicitud de dichos estudios. El resultado de los exámenes solicitados en cuanto a sus valores medios y el desvío estándar se encuentran dentro de los rangos normales para la edad (Tabla 2).

En cuanto a los beneficios de la aplicación de la historia clínica dirigida a la valoración de la hemostasia se evidencio la utilidad en detectar pacientes sospechosos de tener problemas en la hemostasia en el 1,5\% de los casos, sin embargo el uso del screening mediante la solicitud del exámenes complementarios no permitió identificar o descartar casos de trastornos de coagulación, a pesar de que un 21\% (15 casos)

Tabla 1. Resultados de los hallazgos de la historia clinica pediatrica dirigida a la valoración de la hemostasia preoperatoria

\begin{tabular}{|c|c|c|}
\hline \multicolumn{3}{|l|}{ Resultado de la historia clímica pediátrica dirigida a la valoración de la hemostasia preoperatoria } \\
\hline Antecedentes personales de sangrado: & No & Si \\
\hline 1. ¿Ha sido sometido alguna vez a una Cirugía? & 69 & 0 \\
\hline 2. ¿Tuvo sangrado prolongado durante o despues de la Cirugía? & 69 & 0 \\
\hline 3. ¿Requirió transfusiones o motivó prolongación de la hospitalización? & 69 & 0 \\
\hline 4. ¿Ha sido sometido a extracción dental? & 69 & 0 \\
\hline 5. ¿Tuvo sangrado prolongado ( $>10$ minutos) luego de una extracción dental o resangrado al tercer día? & 69 & 0 \\
\hline 6. ¿Presenta equimosis o hematomas frecuentes y/o extensos sin motivo aparente? & 69 & 0 \\
\hline 7. ¿Presentó alguna vez sangrado nasal que requirió tratamiento medico? & 69 & 0 \\
\hline 8. ¿Tarda más de 5 minutos en dejar de sangrar ante una herida cortante o mordedura? & 69 & 0 \\
\hline 9. ¿Ha estado tomando aspirina o analgésicos en la ultima semana? & 69 & 0 \\
\hline 10. ¿Presenta alguna enfermedad por la cual esté en control o tratamiento? ¿Cuál? & 69 & 0 \\
\hline Antecedentes familiares de sangrado: & No & Si \\
\hline 11. ¿Algún pariente ha tenido complicaciones por sangrado en las Cirugía? & 69 & 0 \\
\hline 12 ¿ A lgún pariente presenta hemofilia o von Willebrand? & 69 & 0 \\
\hline Examen físico dirigido: & No & Si \\
\hline 1. Petequias & 69 & 0 \\
\hline 2. Teleangiectasias & 69 & 0 \\
\hline 3. Equimosis & 69 & 0 \\
\hline 4. Hem atom as & 69 & 0 \\
\hline 5. Deformidades articulares & 69 & 0 \\
\hline 6. Hiperlaxitud & 68 & 1 \\
\hline
\end{tabular}

Fuente: elaboración propia 
Tabla 2. Resultados de los exámenes complementarios rutinarios y no rutinarios realizados para la valoración preoperatoria pediátrica

\begin{tabular}{|c|c|c|c|c|c|}
\hline \multirow[b]{3}{*}{ Prueba realizada } & \multicolumn{5}{|c|}{ Resultados de los exámenes complementarios solicitados en la valoración preoperatoria pediátrica } \\
\hline & \multicolumn{5}{|c|}{ Pruebas realizadas rutinariamente a todos los pacientes } \\
\hline & Recuento / Porcentaje & Valor mínimo & Valor maximo & Media & $\begin{array}{c}\text { Desviación } \\
\text { estándar }\end{array}$ \\
\hline Tiempo de protrombina & $69 / 100 \%$ & 10,2 & 20,0 & 13,6 & 1,3 \\
\hline Tiempo parcial de tromboplastina activada & $69 / 100 \%$ & 23,0 & 57,0 & 33,7 & 9,5 \\
\hline Recuento de plaquetas & $69 / 100 \%$ & 174000 & 843000 & 328821 & 118238 \\
\hline Hemoglobina en $\mathrm{mg} / \mathrm{dL}$ & $69 / 100 \%$ & 8,2 & 14,6 & 12,2 & 1,5 \\
\hline \multicolumn{6}{|c|}{ uebas realizadas no rutinariamente (por indicación de cirujano o anestesiólogo) a todos los pacientes } \\
\hline Prueba realizada & Recuento / Porcentaje & Valor mínimo & Valor maximo & Media & $\begin{array}{c}\text { Desviación } \\
\text { Estándar }\end{array}$ \\
\hline Creatinina en $\mathrm{mg} / \mathrm{dl}$ & $33 / 47,8 \%$ & 0,4 & 1,2 & 0,5 & 0,2 \\
\hline Urea serica en $\mathrm{mg} / \mathrm{dl}$ & $25 / 36,2 \%$ & 10,0 & 40,0 & 20,9 & 8,2 \\
\hline Na serico en $\mathrm{mEq} / \mathrm{L}$ & $11 / 15,9 \%$ & 137,0 & 145,0 & 141,7 & 2,5 \\
\hline $\mathrm{K}$ serico en $\mathrm{mEq} / \mathrm{L}$ & $11 / 15,9 \%$ & 3,5 & 5,0 & 4,1 & 0,4 \\
\hline $\mathrm{Cl}$ sérico en $\mathrm{mEq} / \mathrm{L}$ & $10 / 14,5 \%$ & 101,0 & 107,0 & 104,2 & 1,9 \\
\hline Ca sérico en $\mathrm{mg} / \mathrm{dl}$ & $10 / 14,5 \%$ & 1,1 & 1,4 & 1,2 & 0,1 \\
\hline Glicemia sérica en $\mathrm{mg} / \mathrm{dl}$ & $18 / 26,1 \%$ & 67,0 & 125,0 & 97,7 & 15,0 \\
\hline GOT sérico en $\mathrm{mg} / \mathrm{dl}$ & $8 / 11,5 \%$ & 12,0 & 57,0 & 33,8 & 18,0 \\
\hline GPT sérico en $\mathrm{mg} / \mathrm{dl}$ & $8 / 11,5 \%$ & 10,0 & 72,0 & 31,8 & 24,8 \\
\hline Tiempo de Sangria en segundos & $2 / 2,9 \%$ & 1,0 & 2,0 & 1,5 & 0,7 \\
\hline Tiempo de coagulacion en segundos & $13 / 18,8 \%$ & 5,0 & 10,0 & 7,4 & 1,8 \\
\hline
\end{tabular}

Fuente: elaboración propia.

los resultados principalmente de TP estuvieron alterados (Falsos positivos), lo que adicionalmente ocasiono incurrir en conductas para confirmar o corregir los resultados alterados, evidenciándose 4 conductas principales, 1: repetir los estudios alterados 4 casos (6\%), 2: repetir los estudios alterados y administrar vitamina $\mathrm{K} 3$ casos (4\%), 3: administrar vitamina K 7 casos (7\%), 4: transfundir Plasma Fresco Congelado 3 casos (4\%) (Tabla 3).

En cuanto a los costos generados por la realización de exámenes rutinarios a pacientes sometidos a procedimientos menores o ambulatorios, evidenciamos un gasto medio de 47 Bs. por paciente, un gasto adicional promedio de 164 Bs. por exámenes no rutinarios y de 91 Bs. por medidas correctivas o de confirmación ante resultados alterados; con un costo promedio global de 102 Bs. por paciente sometido a procedimientos quirúrgicos menores o ambulatorios (Tabla 3 ).

\section{Discusión}

En la práctica cotidiana continúa siendo ampliamente aceptada la solicitud de exámenes de sangre para realizar un screening de la hemostasia en niños que van a ser sometidos a cirugías menores o ambulatorias ${ }^{10,14}$, siendo que su utilidad es baja en el diagnóstico de coagulopatías severa o en predecir sangrados perioperatorios en niños sanos; por otro lado,

Tabla 3. Descripción de los costos generados en consecuencia de la solicitud de examenes complementarios rutinarios, no rutinarios y de las medidas tomadas para normalizar algun valor de laboratorio anormales.

\begin{tabular}{|c|c|c|c|c|c|}
\hline \multicolumn{2}{|l|}{ Costos analizados } & Recuento & Porcentaje & Costc & (Bs. paciente) \\
\hline \multicolumn{2}{|c|}{ Costo de los exámenes solicitados rutinariamente. } & 69 ptes. & $100 \%$ & & 47 Bs./pte. \\
\hline \multicolumn{2}{|c|}{$\begin{array}{l}\text { Costo de los exámenes no rutinariarios según criterio de } \\
\text { cirujano o anestesiólogo }\end{array}$} & 15 ptes. & $21,7 \%$ & & 164 Bs./pte. \\
\hline \multirow{6}{*}{$\begin{array}{c}\text { Costo de las medidas tomadas para } \\
\text { normalizar algún valor de laboratorio } \\
\text { anormal. }\end{array}$} & Ninguno & 54 ptes. & $78 \%$ & $0 \mathrm{Bs}$. & \multirow{6}{*}{91,2 Bs./pte. } \\
\hline & $\begin{array}{l}\text { Repetir estudio o estudios } \\
\text { alterados TP y/ APTT }\end{array}$ & 4 ptes. & $6 \%$ & $47 \mathrm{Bs}$. & \\
\hline & $\begin{array}{l}\text { Repetir estudio y administrar } \\
\text { vitamina K } 3 \text { dosis }\end{array}$ & 3 ptes. & $4 \%$ & $67 \mathrm{Bs}$. & \\
\hline & Administrar vitamina $\mathrm{K}$ & 5 ptes. & $7 \%$ & $20 \mathrm{Bs}$. & \\
\hline & $\begin{array}{l}\text { Transfusion de plasma fresco } \\
\text { congelado }\end{array}$ & 2 ptes. & $3 \%$ & 264 Bs. & \\
\hline & $\begin{array}{l}\text { Repetir estudio, Administrar } \\
\text { vitamina K y transfundir PFC } 2\end{array}$ & 1 ptes. & $1 \%$ & $351 \mathrm{Bs}$. & \\
\hline
\end{tabular}

Fuente: elaboración propia 
siendo su utilidad indiscutible en paciente con anticoagulación, o en pacientes con deficiencias en factores de coagulación conocidas preoperatorialmente.

Los hallazgos clínicos y el examen físico dirigido a la valoración de la hemostasia identificaron un solo paciente con hallazgo positivo de sospecha de coagulopatía, el cual fue descartado posteriormente, sin observarse complicaciones intra o postoperatorias en la serie de casos estudiados; resultados similares se evidenciaron en un estudio con 56000 cirugías, de las cuales 5120 se realizaron sin estudios preliminares y solo con la exploración clínica dirigida normal, se evidencio que los pacientes pudieron someterse a la anestesia y cirugía de forma segura y con buenos resultados intra y postoperatorios ${ }^{6}$. Otro estudio realizado en 169 adultos sometidos a herniorrafias permite concluir que es raro que una historia clínica y examen físico normales presenten sangrados perioperatorios, sugiriendo que los test de rutina preoperatoria en pacientes sanos es de poco valor?.

A pesar de la evidencia disponible, la rutina preoperatoria en muchos centros quirúrgicos pediátricos incluye un screening laboratorial de la hemostasia, los test más solicitados son el TP, APTT y hemograma, aunque también observamos pruebas como el tiempo de sangría y el tiempo de coagulación; en nuestro estudio de 69 estudios rutinarios observamos alteración en el $21 \%$ de ellos, principalmente en el TP, sin embargo no observamos complicaciones hemorrágicas intra o postoperatorias, ni se diagnosticó algún trastorno de coagulación en los niños con pruebas alteradas. Lo que se explica en base a los hallazgos de un metanálisis que evaluó 9 estudios observacionales de buena calidad, corroborando que los test de coagulación tienen un valor predictivo positivo $(0,03-0,22)$ y un likelihood ratio $(0,94-5,1)$ bajos que indican que son predictores pobres de sangrado, y recomendando que los pacientes con historia clínica negativa a sangrados no requieren de screening laboratorial previo a la cirugía ${ }^{2}$.

El estudio también aporta información sobre los test solicitados de forma arbitraria (no de rutina) y sobre algunas medidas tomadas en cuenta para corregir algún resultado alterado en los test de rutina; observamos que 149 test (de 218 test) fueron solicitados no de rutina y sin justificación clínica, sin aportar beneficio en cuanto al manejo clínico o quirúrgico; resultados similares se analizan en 2 estudios en 520 pacientes sometidos a cirugías no cardiacas, en los cuales se concluye que los exámenes rutinarios no son ni útiles ni costo-efectivos, recomendando que sean solicitados solo en base a las enfermedades concomitantes, tipo de cirugía a realizar $^{5,7}$.

Algo no descrito en literatura similar es la práctica de corregir valores de algún examen complementario alterado, sin corroborar con la clínica, practica identificada en el 18\% de los pacientes y que incluye desde repetir y confirmar el estudio alterado a la transfusión de hemoderivados, situación sobremanera llamativa, porque no solo podría considerarse sin utilidad y no costo-efectiva, sino también riesgosa por las eventuales complicaciones de la trasfusión de hemoderivados, que en estos casos superan por mucho a los beneficios; para los fines del estudio no se realizó seguimiento a los pacientes transfundidos, sin embargo ninguno presento complicaciones durante su estancia hospitalaria.

El beneficio de un screening laboratorial para trastornos de la coagulación con los test es baja, principalmente por su baja sensibilidad y especificidad, así también como su bajo valor predictivo positivo y likelyhood ratio $^{2}$, adicionalmente a esto mencionar que los trastornos de la coagulación son enfermedades poco frecuentes; en el HNMAV el año 2018 de 55000 pacientes atendidos se diagnosticaron 2 casos de hemofilia al año principalmente por clínica, no se diagnosticaron otras coagulopatías en dicho año.

Los cargos a los pacientes ascendieron a 47 Bs por paciente de forma rutinaria, y de 255 Bs por paciente por cargos asociados a pruebas no rutinarias o destinados a la corrección de valores alterados en 15 pacientes; por lo que en término medio se eroga un costo en pruebas de laboratorio preoperatorios de aproximadamente 102 Bs por paciente.

$\mathrm{Si}$ las pruebas de laboratorio para todos los pacientes ambulatorios de cirugía o para procedimientos menores se hubieran ordenado según los hallazgos del historial médico y el examen físico del paciente en lugar de hacerlo por criterios de rutina o arbitrarios, en 69 pacientes estudiados solamente se justificaba las pruebas laboratorial en 1 caso; por lo que nuestro hospital podría haber reducido potencialmente los costos en los paciente en más de 7000 Bs. en el grupo de estudio, y se puede inferir que durante el periodo de un año se puede reducir entre 32100 Bs. a 187000 Bs. sin resultados adversos esperados.

En conclusión, el estudio estableció que las pruebas rutinarias de screening preoperatorio tienen poca utilidad y son poco costo-beneficiosas en la valoración de la hemostasia para procedimientos menores o ambulatorios, en comparación de una historia clínica y examen físico dirigido; siendo apropiada su indicación cuando existan hallazgos anormales en el examen físico e historia clínica o en base a enfermedades concomitantes; estudios multicentricos adicionales son requeridos ${ }^{16}$ para extender las recomendaciones a otras ciudades de las mismas características geográficas, de altitud a nivel del mar y sociodemográficas de nuestro país, procurando guiar las conductas medicas a ser más eficientes con el uso de los recursos, en un contexto de recursos limitados.

Conflicto de intereses: los autores declaran que no existe conflicto de intereses. 


\section{Referencias bibliográficas}

1. Rapaport SI. Preoperative hemostatic evaluation: which tests, if any? Blood 1983. https://doi.org/10.1182/blood.V61.2.229. bloodjournal612229

2. Chee YL, Crawford JC, Watson HG, Greaves $\mathrm{M}$. Guidelines on the assessment of bleeding risk prior to surgery or invasive procedures. British Committee for Standards in Haematology. Br J Haematol 2008.

https://doi.org/10.1111/j.1365-2141.2007.06968.x

3. Cosmi B, Alatri A, Cattaneo M, et al. Assessment of the risk of bleeding in patients undergoing surgery or invasive procedures: Guidelines of the Italian Society for Haemostasis and Thrombosis (SISET). Thromb Res 2009.

https://doi.org/10.1016/j.thromres.2009.08.005

4. De Paepe A, Malfait F. Bleeding and bruising in patients with Ehlers-Danlos syndrome and other collagen vascular disorders. Br J Haematol 2004.

https://doi.org/10.3109/9780203997352.078

5. Velanovich V. The value of routine preoperative laboratory testing in predicting postoperative complications: a multivariate analysis. Surgery 1991.

6. Narr BJ, Warner ME, Schroeder DR, Warner MA. Outcomes of patients with no laboratory assessment before anesthesia and a surgical procedure. Mayo Clin Proc 1997.

https://doi.org/10.1016/S0002-9394(14)70851-7

7. Velanovich V. Preoperative laboratory screening based on age, gender, and concomitant medical diseases. Surgery 1994.

https://doi.org/10.1097/00132586-19941000000026

8. Wattsman TA, Davies RS. The utility of preoperative laboratory testing in general surgery patients for outpatient procedures. Am Surg 1997.

9. Adams JG Jr, Weigelt JA, Poulos E. Usefulness of preoperative laboratory assessment of patients undergoing elective herniorrhaphy. Arch Surg 1992. https://doi.org/10.1001/ archsurg.1992.01420070061012

10. Silver D. What Routine Preoperative Tests for Bleeding Tendencies? JAMA 1976.

11. Eisenberg JM, Clarke JR, Sussman SA. Prothrombin and partial thromboplastin times as preoperative screening tests. Arch Surg 1982. https://doi.org/10.1001/ archsurg.1982.01380250030007

12. Kaplan EB, Sheiner LB, Boeckmann AJ, et al. The usefulness of preoperative laboratory screening. JAMA 1985. https://doi.org/10.1001/ jama.1985.03350480084025
13. Segal JB, Dzik WH, Transfusion Medicine/ Hemostasis Clinical Trials Network. Paucity of studies to support that abnormal coagulation test results predict bleeding in the setting of invasive procedures: an evidence-based review. Transfusion 2005.

https://doi.org/10.1111/j.1537-2995.2005.00546.x

14. Wahlberg T, Blombäck M, Hall P, Axelsson G. Application of indicators, predictors and diagnostic indices in coagulation disorders. I. Evaluation of a self-administered questionnaire with binary questions. Methods Inf Med 1980. https://doi.org/10.1055/s-0038-1635279.

15. Laine C, Williams SV, Wilson JF. In the clinic. Preoperative evaluation. Ann Intern Med 2009.

https://doi.org/10.7326/0003-4819-151-1200907070-01006

16. Copana R, Melean LG. Hiponatremia en pacientes postoperados en el Hospital del Niño Manuel Ascencio Villarroel, Cochabamba, Bolivia. Gac Med Bol. 2014. 